\title{
Vacinação contra o Papiloma Vírus Humano no Brasil: uma interlocução com as publicações científicas
}

Vaccination against human papilloma virus in Brazil: a dialogue with the scientific publications

La vacunación contra el Virus del Papiloma Humano en Brasil: un diálogo con las publicaciones científicas

\section{Ananda Kauanne Costa da Silva ${ }^{1}$; José de Ribamar Ross ${ }^{2}$}

\section{Resumo}

O Papiloma Vírus Humano (HPV) é um agente infeccioso de transmissão sexual, e uma das infecções mais comum mundialmente, sendo mais susceptível na adolescência e na fase adulta. $\mathrm{O}$ estudo objetivou investigar a eficácia das vacinas profiláticas disponíveis contra o Papiloma Vírus Humano para a redução da incidência do câncer do colo do útero. Trata-se de uma pesquisa de revisão integrativa, de caráter exploratório-descritivo, com abordagem quantitativa. Os resultados dos estudos mostraram que a vacina Papiloma Vírus Humano proporciona a diminuição considerável das infecções referentes aos sorotipos encontrados na vacina, bem como a diminuição do aparecimento das verrugas genitais e lesões cervicais de alto grau. Além disso, a administração da vacina em 50\% das adolescentes da cidade de Mali, África Ocidental diminuiu a predominância dos sorotipos para 5\% na zona urbana e 9,6\% na zona rural. Ademais a vacina reduziu o índice de mortalidade ocasionada pela neoplasia uterina, que provocou a morte de 1.145 na zona urbana e 2.742 na zona rural. É necessário que haja interesse dos pesquisadores brasileiros em pesquisar o impacto das vacinas para a prevenção do câncer do colo do útero, em virtude do seu pouco tempo de uso como principal medida preventiva

Descritores: Vacinação de adolescente. Programas de imunização Calendário de vacinação das adolescentes. Vacinação quadrivalente recombinante contra HPV tipos 6,11, 16, 18. Vacinas contra papillomavírus

\footnotetext{
Abstract

The Human Papilloma Virus (HPV) is an infectious agent of sexual transmission, and one of the most common infections worldwide, more likely in adolescence and adulthood. The study aimed to investigate the effectiveness of prophylactic vaccines available against the Human Papilloma Virus to reduce the incidence of cervical cancer. This is an integrative review of research, exploratory and descriptive, with a quantitative approach. The results of the studies showed that the Human Papilloma Virus vaccine provides significant reduction of infections related to serotypes found in the vaccine, as well as decreasing the onset of genital warts and

${ }^{1}$ Graduação em Enfermagem pela Faculdade de Ciência e Tecnologia do Maranhão (FACEMA). Estagiária do Serviço de Vigilância Epidemiológica da Secretaria de Saúde de Caxias. Praça Dias Carneiro, 600, Centro, CEP: 65.604-090 - Caxias - MA. Email: kauanne_nanda@hotmail.com

${ }^{2}$ Mestre em Enfermagem pela Universidade do Vale do Rio Sinos (UNISINOS). Professor da Universidade Estadual do Maranhão, Centro de Estudos Superiores de Caxias. Rua Quininha Pires S/N Centro. 65600-310 Caxias, MA - Brasil. Email: enfross@hotmail.com
} 
ISSN 2179-6750

cervical high-grade lesions. In addition, the administration of the vaccine in $50 \%$ of adolescents in the city of Mali, West Africa decreased the prevalence of serotypes to $5 \%$ in urban areas and $9.6 \%$ in rural areas. In addition the vaccine reduced the mortality rate caused by uterine cancer, which caused the death of 1,145 in the urban area and 2,742 in the countryside. There must be interest of Brazilian researchers in researching the impact of vaccines for the prevention of cervical cancer, because of their short time of use as a primary preventive measure.

Key-words: Adolescent vaccination. Immunization programs. Of adolescent vaccination schedule. Recombinant quadrivalent vaccine against HPV types 6, 11, 16, 18 e Papillomavirus vaccine.

\section{Resumen}

El Virus del Papiloma Humano (VPH) es un agente infeccioso de transmisión sexual, y una de las infecciones más comunes en todo el mundo, más probables en la adolescencia y la edad adulta. El objetivo del estudio fue investigar la eficacia de las vacunas profilácticas disponibles contra el virus del papiloma humano para reducir la incidencia de cáncer de cuello uterino. Se trata de una revisión integradora de la investigación, exploratorio y descriptivo, con enfoque cuantitativo. Los resultados de los estudios mostraron que la vacuna del virus del papiloma humano proporciona una reducción significativa de las infecciones relacionadas con los serotipos encontrados en la vacuna, así como la disminución de la aparición de las verrugas genitales y lesiones de alto grado cervicales. Además, la administración de la vacuna en el $50 \%$ de los adolescentes en la ciudad de Mali, África Occidental disminuyó la prevalencia de los serotipos de 5\% en las zonas urbanas y el 9,6\% en las zonas rurales. Además, la vacuna redujo la tasa de mortalidad causada por el cáncer de útero, lo que causó la muerte de 1.145 en el área urbana y 2.742 en el campo. Tiene que haber interés de los investigadores brasileños en la investigación de los efectos de las vacunas para la prevención de cáncer de cuello uterino, debido a su corto tiempo de uso como medida de prevención primaria.

Palabras-claves: La vacunación de los adolescentes. Los programas de inmunización para el calendario de vacunación de los adolescentes. Vacuna tetravalente recombinante contra los tipos de VPH 6, 11, 16, 18. Vacuna tetravalente recombinante contra los tipos de VPH 6, 11, $16,18$.

\section{Introdução}

O Papiloma Vírus Humano (HPV) é um agente infeccioso de transmissão sexual, e uma das infecções mais comum mundialmente, sendo mais susceptível na adolescência e na fase adulta ${ }^{1}$. No Brasil, o câncer do colo do útero é o terceiro mais prevalente em mulheres, perdendo somente para o câncer de pele não melanoma e câncer de mama, é também a segunda razão de óbitos por câncer entre a população feminina ${ }^{2}$.

No ano de 2006 foi aprovada a primeira vacina preventiva contra o Papiloma Vírus Humano pelo Food and Aministration para os sorotipos 6, 11, 16 e 18 (quadrivalente) ${ }^{3}$. Em 
ISSN 2179-6750

2008 foi autorizada a segunda vacina, porém esta apenas prevenirá contra os sorotipos 16 e 18 (bivalente). Estas vacinas profiláticas foram inicialmente disponibilizadas para a população brasileira até o ano de 2013 apenas para os serviços privados de assistência à saúde ${ }^{4}$.

As duas vacinas preventivas foram legalizadas no Brasil pela Agência Nacional de Vigilância Sanitária (ANVISA). No ano de 2014, o Ministério da Saúde introduziu a vacina quadrivalente contra o HPV gratuitamente no SUS para as meninas com faixa etária entre 9 a 13 anos, já que a infecção pelo vírus é mínima nessa faixa etária e também por haver eficiente fornecimento de anticorpos ${ }^{5}$. Apenas a vacina quadrivalente $(6,11,16$ e 18$)$ em combate ao vírus Papiloma Humano foi introduzida no serviço público de Saúde pela portaria ${ }^{\circ}$ 54, de 18 de novembro de 2013, com o objetivo é prevenir o desenvolvimento da neoplasia cervical ${ }^{6}$.

Diante disso, buscou-se investigar a eficácia das vacinas profiláticas disponíveis contra o Papiloma Vírus Humano para a redução da incidência do câncer do colo do útero. Além de verificar a ocorrência de eventos adversos pós-vacinação, bem como as contraindicações para sua administração.

\section{Métodos}

Trata-se de uma pesquisa de revisão integrativa, de caráter exploratório-descritivo, com abordagem quantitativa. Foi realizada coleta de artigos na Biblioteca Virtual em Saúde BVS, nas bases indexadas: SCIELO, LILACS e MEDLINE, sendo a coleta realizada pela própria autora entre os dias 15 e 18 de setembro.

Para a coleta de dados foram utilizados os seguintes descritores: vacinação de adolescente, programas de imunização, calendário de vacinação das adolescentes, vacinação quadrivalente recombinante contra HPV tipos 6, 11, 16, 18 e vacinas contra papillomavírus. Através da pesquisa booleana os descritores foram organizados da seguinte maneira: vacinação de adolescente or programas de imunização or calendário de vacinação dos adolescentes 18 or vacinas contra papillomavírus and vacinação quadrivalente recombinante contra HPV tipos 6, 11, 16, 18.

Foram utilizados os seguintes critérios de inclusão: artigos de língua inglesa, completos, publicados de 2010 a 2015, de exclusão: teses, dissertações, monografia e relatos de experiências, pesquisas qualitativas, revisões e manuais.

Após coleta dos artigos os mesmos foram catalogados, tabulados e apresentados as características dos mesmos em quadro segundo: autor, ano, título, objetivo, metodologia, base 
ISSN 2179-6750

coletada, revista. Os dados foram discutidos de acordo com a literatura e os objetivos propostos.

\section{Resultados}

O presente estudo considerou para extração e análise 35 publicações, nos quais abordavam assuntos pertinentes às discussões envolvidas com a temática. Após refinada a pesquisa e de acordo com os critérios de inclusão e exclusão, foram selecionados 05 artigos para a construção da temática. De acordo com o quadro 01 a base de dados Medline foi utilizada $100 \%$, com isso fica evidenciado que essa fonte concedeu mais publicações sobre o tema eficácia da vacina HPV, concedendo o total de 05 publicações. Com base na análise dos periódicos, percebe-se que as revistas Vaccine e Clinical Therapeutics proporcionaram o maior número de publicações concedendo cada uma, total de 02 publicações. Entretanto, a revista Pharmaco epidemiology and Drug Safet, proporcionou o menor número de artigos publicados concedendo apenas 01 publicações. Conforme o ano de publicação dos estudos, percebe-se que o ano de 2014 concedeu maior número de publicações para a amostra, o idioma inglês foi utilizado $100 \%$ na pesquisa demonstrando o déficit de estudos no Brasil sobre a eficácia da vacinação contra HPV entre as adolescentes.

\section{Quadro 1. Quadro demonstrativo de operadores de busca na BVS}

\begin{tabular}{|l|c|}
\hline \multicolumn{1}{|c|}{ PROBLEMA } & TOTAL \\
\hline $\begin{array}{l}\text { (tw:(vacinação de adolescente)) OR (tw:(programas de imunização)) } \\
\text { OR (tw:(calendário de vacinação do adolescentes)) AND } \\
\text { (instance:"regional") }\end{array}$ & (9377) \\
\hline \multicolumn{1}{|c|}{ INTERVENÇÃ O } & \\
\hline $\begin{array}{l}\text { (tw:(vacinação quadrivalente recombinante contra hpv tipos 6, 11, 16, } \\
\text { 18)) OR (tw:(vacinas contra papillomavírus)) AND } \\
\text { (instance:"regional") }\end{array}$ & (3828) \\
\hline $\begin{array}{l}\text { (tw:((tw:(vacinação de adolescente)) OR (tw:(programas de } \\
\text { imunização)) OR (tw:(calendário de vacinação do adolescentes)) }\end{array}$ & $(211)$ \\
\hline \multicolumn{2}{|c|}{ DISPONIVEL MEDLINE 2014 } \\
\hline Fonte: BVS, 2016
\end{tabular}

\section{Discussão}

Segundo Brasil ${ }^{7}$, a vacinação contra o HPV não deve ser administrada nas 94 
ISSN 2179-6750

adolescentes com hipersensibilidade a substância ou a outro componente da vacina, com história de hipersensibilidade direta danosa à levedura e que após a vacinação apresentem sintomas que indicam hipersensibilidade danosa. Além disso, mulheres grávidas também não poderão receber as doses da vacina, já que até o momento não há pesquisas concludentes.

Segundo o mesmo autor nos casos em que ocorra a gestação após iniciar as doses da vacina, a dose seguinte deverá ser prorrogada até o nascimento do bebê ou quando a administração ocorrer desavisadamente na gestação, a única intervenção será o acompanhamento adequado do pré-natal. Após análise dos artigos selecionados para a pesquisa, percebe-se que ainda não foram realizados estudos relacionados sobre a ocorrência das contraindicações para a administração das vacinas profiláticas contra o vírus Papiloma Humano, devido à dificuldade de se encontrar estudos que abordassem tal temática, sendo encontrado no Brasil, apenas nos manuais do Ministério da Saúde.

Segundo o experimento realizado na Austrália com a vacina Papiloma Vírus Humano, a mesma proporcionou a diminuição considerável das infecções referentes aos sorotipos encontrados na vacina, bem como a diminuição do aparecimento das verrugas genitais e lesões cervicais de alto grau ${ }^{8}$.

Uma pesquisa realizada na cidade de Mali, África Ocidental com o intuito de avaliar a repercussão e a eficácia de um programa que tem como foco o combate ao vírus HPV, demonstrou que a administração da vacina em 50\% das adolescentes diminuiu a predominância dos sorotipos para 5\% na zona urbana e 9,6\% na zona rural. Além disso, a vacina reduziu o índice de mortalidade ocasionada pela neoplasia uterina, que provocou a morte de 1.145 na zona urbana e 2.742 na zona rural ${ }^{9}$.

A administração da vacina preventiva contra o HPV tem demonstrado ser uma estratégia fundamental para a diminuição do câncer do colo do útero. No entanto, a administração da vacina tem demonstrado maior eficácia quando administrada nas adolescentes na faixa etária entre 9 a 13 anos de idade que ainda não tiveram relação sexual, ou seja, não entraram em contato com o vírus HPV ${ }^{10}$.

Após administração da vacina quadrivalente nas adolescentes, foram analisados dados sobre o aparecimento de eventos adversos após a vacinação, sendo os mais frequentes a reação alérgica e no local da aplicação e erupção cutânea. Além disso, foram relatados a ocorrência de outros eventos graves como o aparecimento de casos de anafilaxia, convulsão, trombocitopenia e morte. Entretanto, foi constatado que os casos de anafilaxia não 
ISSN 2179-6750

correspondem a anafilaxia Brigton e o óbito foi ocasionado por um problema cardíaco que já existia anteriormente ${ }^{11}$.

No Reino Unido foram relatados casos de eventos adversos após a vacinação, o que ocasionou uma reavaliação sobre a ocorrência da anafilaxia, angioedema e síncope. Um estudo realizado com 4.643 mulheres que foram vacinadas, não foi detectado a ocorrência de eventos adversos graves, como também não foram detectados obstáculos quanto a seguridade da vacina. Entretanto, o falecimento precoce de um jovem no Reino Unido após ter sido vacinado, chamou a atenção dos meios de comunicação, mas após averiguação, não foram encontradas quaisquer falhas da vacina e os resultados após a morte do adolescente, embora tenha sido diagnosticado inicialmente com uma provável reação anafilática foi comprovado que o mesmo sofria de doença rara ${ }^{12}$.

A vacinação tanto com a bivalente quanto com a quadrivalente não oferecem perigo, além disso, geram resposta imunológica e apresentam bastante eficiência para prevenir o contato frequente, com as formas contidas em ambas as vacinas, assim como ajudam a prevenir contra as lesões que tem potencial para progredir diretamente para a neoplasia invasiva $^{13}$.

Observa-se que apesar dos relatos da ocorrência de eventos adversos após a vacinação das adolescentes, não foram encontrados estudos que comprovassem tais ocorrências, demonstrando que as vacinas, são na verdade uma estratégia de prevenção eficaz no combate a transmissão do vírus HPV.

\section{Conclusão}

As vacinas preventivas foram aprovadas no Brasil recentemente com o objetivo de prevenir o aparecimento das lesões precursoras que desencadeiam o câncer do colo do útero. Entretanto, ainda existem poucos estudos que avaliam a eficácia dessas vacinas. Já no Brasil, os estudos sobre tal eficiência ainda são escassos, como pode ser comprovado pela pesquisa, devido à dificuldade de se encontrar estudos relacionados com a temática.

Almeja-se com este estudo, contribuir para o esclarecimento sobre a eficácia da vacinação, além de incentivar o interesse por parte dos pesquisadores brasileiros para a produção de mais estudos sobre a temática. Diante disso, é de suma importância a realização de pesquisas sobre o impacto das vacinas para a prevenção do câncer do colo do útero, em virtude do pouco tempo de uso dessas vacinas como principal medida preventiva. 


\section{Referências}

1. Rodrigues DA, Pereira ER, Oliveira LSS, Speck NMG, Gimeno SGA. Prevalência de atipias citológicas e infecção pelo papilomavírus humano de alto risco em mulheres indígenas Panará, povo indígena do Brasil Central. Cad Saúde Pública [periódico na Internet]. 2014 Dez [acessado 2015 Mar 15]; 30(12): [cerca de 7 p.]. Disponível em: http://www.scielosp.org/pdf/csp/v30n12/0102-311X-csp-30-12-02587.pdf.

2. Pinto DS, Fuzii HT, Quaresma JAS. Prevalência de infecção genital pelo HPV em populações urbana e rural da Amazônia Oriental Brasileira. Cad Saúde Pública [periódico na Internet]. 2011 Abr [acessado 2015 Set 16]; 27(4): [cerca de 10 p.]. Disponível em: http://www.scielo.br/pdf/csp/v27n4/16.pdf.

3. Borsatto AZ, Vidal MLB, Rocha RCNP. Vacina contra o HPV e a Prevenção do Câncer do Colo do Útero: Subsídios para a Prática. Rev Bras Cancerol [periódico na Internet]. 2011[acessado 2015 Set 18]; 57(1): [cerca de 8 p.]. Disponível em: http://www1.inca.gov.br/rbc/n_57/v01/pdf/10_revisao_de_literatura_vacina_hpv_prev encao_cancer_colo_utero_subsidios.pdf.

4. Osis MJD, Duarte GA, Sousa MH. Conhecimento e atitude de usuários do SUS sobre o HPV e as vacinas disponíveis no Brasil. Rev Saúde Pública [periódico na internet]. 2014 [acessado 2015 Set 17]; 48(1): [cerca de 11 p.]. Disponível em: http://www.scielo.br/pdf/rsp/v48n1/0034-8910-rsp-48-01-0123.pdf.

5. BRASIL. Instituto Nacional de Câncer. HPV e câncer - Perguntas mais freqüentes. 2016. Disponível em: http://www1.inca.gov.br/conteudo_view.asp?id=2687. Acesso em: 15 set. 2015.

6. BRASIL. Ministério da Saúde. Portaria n ${ }^{\circ} 54$ de 18 de janeiro de 2013. Torna pública a decisão incorporar a vacina quadrivalente contra HPV na prevenção do câncer de colo do útero no Sistema Único de Saúde - SUS, 2013. Disponível em: http://bvsms.saude.gov.br/bvs/saudelegis/sctie/2013/prt0054_18_11_2013.html.

Acesso em: 16 set. 2015.

7. BRASIL. Ministério da Saúde. Guia de perguntas e respostas para Profissional de Saúde. 2014.

Disponível em: http://portalsaude.saude.gov.br/images/pdf/2014/marco/07/guia-perguntas-repostasMS-HPV-profissionais-saude2.pdf. Acesso em 16 set. 2015. 
ISSN 2179-6750

8. Garland SM. The Australian Experience With the Human Papillomavirus Vaccine. Clinical Therapeutics [periódico na internet]. 2014 Jan [acessado 2015 Set 16]; 36(1): [cerca de 9 p.]. Disponível em: http://www.clinicaltherapeutics.com/article/S01492918(13)01120-X/pdf.

9. Tracy JK, Schluterman NH, Greene C, Sow SO, Gaff HD. Vaccine [periódico na internet]. 2014 May [acessado 2015 Set 17]; 32(26): [cerca de 6 p.]. Disponível em: http://www.sciencedirect.com/science?_ob=ShoppingCartURL\&_method=add\&_eid= $1-\mathrm{s} 2.0$

S0264410X14004368\&originContentFamily=serial\&_origin=article\&_ts=146728725 2\&md5=63932f319d69ae540ba0506363e123ee.

10. Erickson BK, Landers EE, Huh WK. Update on Vaccination Clinical Trials for HPVRelated Disease. Clinical Therapeutics [periódico na internet]. 2014 [acessado 2015 Set 16]; 36(1): [ cerca de 9 p.]. Disponível em: http://www.clinicaltherapeutics.com/article/S0149-2918(13)01079-5/pdf.

11. Harris T, Williams DM, Fediurek J, Scott T, Deeks SL. Adverse events following immunization in Ontario's female school-based HPV program. Vaccine [periódico na internet]. 2014 Feb [acessado 2015 Set 15]; 32(9): [cerca de 6p.]. Disponível em: http://www.sciencedirect.com/science/article/pii/S0264410X1400005X.

12. Angelo MG, Zima J, Silva FT, Baril L, Arellano F. Post-licensure safety surveillance for human papillomavirus-16/18-AS04-adjuvanted vaccine: more than 4 years of experience. Pharmaco epidemiology and Drug Safet [periódico na internet]. 2014 [acessado 2015 set 17]; 23: [cerca de 10 p.]. Disponível em: http://www.ncbi.nlm.nih.gov/pmc/articles/PMC4265196/pdf/pds0023-0456.pdf.

13. Limberger, A et al. Aspectos imunológicos da infecção pelo vírus do papiloma humano (HPV). Semina: Ciências Biológicas e da Saúde. [periódico na internet]. 2012 Jan-Jun [acessado 2015 set 18]; 33(1): [cerca de 12 p.]. Disponível em: http://www.uel.br/revistas/uel/index.php/seminabio/article/view/9917/11075. 
